Laser Chem., 1999, Vol. 19, pp. 153-159

Reprints available directly from the publisher Photocopying permitted by license only
(C) 1999 OPA (Overseas Publishers Association) N.V. Published by license under the Harwood Academic Publishers imprint, part of The Gordon and Breach Publishing Group.

\title{
TUNABLE PICOSECOND OPTICAL PARAMETRIC AMPLIFIERS FOR TIME RESOLVED RESONANCE RAMAN SPECTROSCOPY
}

\author{
M. TOWRIE ${ }^{\mathrm{a}, *}$, G. GABOREL ${ }^{\mathrm{a}}$, P. MATOUSEK ${ }^{\mathrm{a}}$, \\ A. W. PARKER ${ }^{\mathrm{a}}$, W. SHAIKH ${ }^{\mathrm{a}}$ and R. H. BISBY ${ }^{\mathrm{b}}$ \\ ${ }^{a}$ LSF, Central Laser Facility, Rutherford Appleton Laboratory, Chilton, \\ Didcot, Oxfordshire, OX11 0QX, UK, \\ ${ }^{\mathrm{b}}$ Department of Biological Sciences, University of Salford, \\ Salford, M5 4WT
}

(Received 30 April 1997)

\begin{abstract}
A picosecond optical parametric amplifier, OPA, system suitable for pump and probe spectroscopy has been developed. Two OPAs are simultaneously pumped by a titanium sapphire regenerative amplifier to produce several microjoules output over the 470 to $2000 \mathrm{~nm}$ wavelength range. One OPA used as the probe operates with c. $25 \mathrm{~cm}^{-1}$ linewidth over this tuning range. The system is optimised for $\mathrm{TR}^{3}$ spectroscopy. Some preliminary experiments on Trolox, a water soluable Vitamin E derivative, are presented.
\end{abstract}

Keywords: Optical parametric amplifier; OPA; picosecond; time resolved; Raman; $\mathrm{TR}^{3}$

\section{INTRODUCTION}

Many ultrafast time-resolved techniques require two broadly and independently tunable, synchronised light pulses. Recent advances in short pulse laser technology and non-linear crystal materials such as $\mathrm{BBO}$ have encouraged the rapid development of optical parametric oscillators (OPOs) and traveling wave optical parametric amplifiers (OPAs) capable of meeting the needs of ultrafast spectroscopists. We

\footnotetext{
* Corresponding author.
} 
have developed kilohertz repetition rate OPA systems providing a stable, high repetition rate, high spectral brightness laser source tunable through the UV and visible wavelengths [1]. The system is optimised for femtosecond/picosecond, pump and probe time-resolved absorption, reflectance and resonance Raman spectroscopies.

\section{Description of the System}

Each OPA is based, see Figure 1, on a Type I BBO $\left(5 \mathrm{~mm}, 2^{\circ}\right)$ crystal which is pumped by a single titanium sapphire regenerative amplifier operating at $1 \mathrm{kHz}$ repetition rate around $800 \mathrm{~nm}, 600-800 \mu \mathrm{J}$ pulse energy and $\sim 1$ ps pulse width. A three stage pump arrangement is used. The first stage generates seed light by optical parametric generation, OPG, ('superfluorescence' generation). A $60 \mathrm{~cm}$ lens focuses a $\sim 20 \mu \mathrm{J}$ split of $400 \mathrm{~nm}$ pump light into the BBO crystal. Tilting this lens creates an aberrated focus elongated in the direction of angle walk-off between pump signal and idler within the crystal. Since the total gain is a stronger function of effective crystal length than of pump intensity this enhances the OPG process. For the second stage the same pump beam and the OPG light are retroreflected back to the crystal. The pump beam is refocused into the crystal. Group velocity mismatch accrued in the OPG stage is compensated by a 'magic' mirror. The output from this stage is a beam with several 10's of $n J$ of energy and provides good quality seed light for the final amplifier stage. Both OPAs use this configuration but OPA 1 uses a grating dispersion element to spectrally filter the pulse prior to injection into

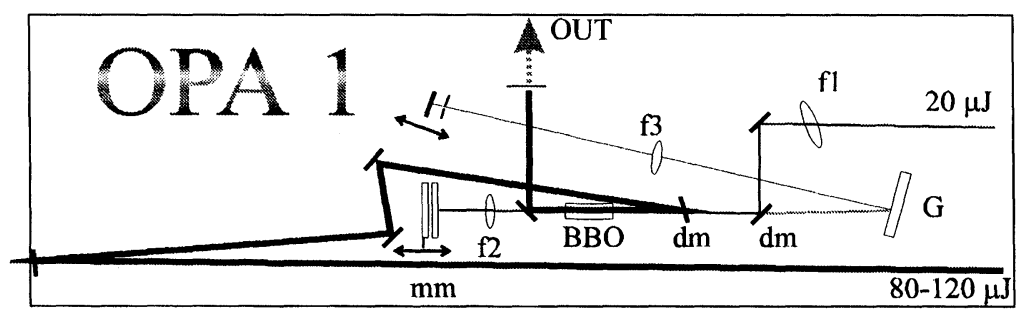

FIGURE 1 OPA $1 . \mathbf{B B O}=29^{\circ}$ cut Type I $5 \mathrm{~mm}$ long BBO crystal, $\mathbf{f 1}, \mathbf{f}, \mathbf{f 3}=60,20$ and $50 \mathrm{~cm}$ lens respectively, $\mathrm{mm}=$ mirror compensating for group velocity mismatch, $\mathbf{d m}=$ dichroic mirrors to reflect pump and transmit signal, $\mathbf{G}=$ Grating, $\mathbf{S}=$ Slit. 
the final amplification stage. This also reconstitutes the beam with no spatial chirp before final amplification and lifts the seed beam in the vertical plane so that the amplified light can be picked off.

For the last stage the pump beam energy in OPA1 is typically $80-120 \mu \mathrm{J}$ and in OPA2 $60-80 \mu \mathrm{J}$. Care is taken to maintain good spatial beam quality in the pump and to minimise beam divergence because these have adverse effects on efficiency and linewidth. The overall pump to signal conversion efficiency of OPA1 and OPA2 is $10 \%$ and $15 \%$ respectively.

Typical performance curves for the OPAs is shown in Figure 2. Using both OPAs and a combination of non-linear conversion processes continuous tuning from $220-2200 \mathrm{~nm}$ at $>0.1-1 \mu \mathrm{J}$ at the sample is possible. We estimate the spatial beam quality to be $<2$ times diffraction limited and the time bandwidth product 1.8 times that of a Gaussian pulse. Shot to shot stability is around $\pm 10 \%$ but constant output is routinely maintained over 10 hours or more.

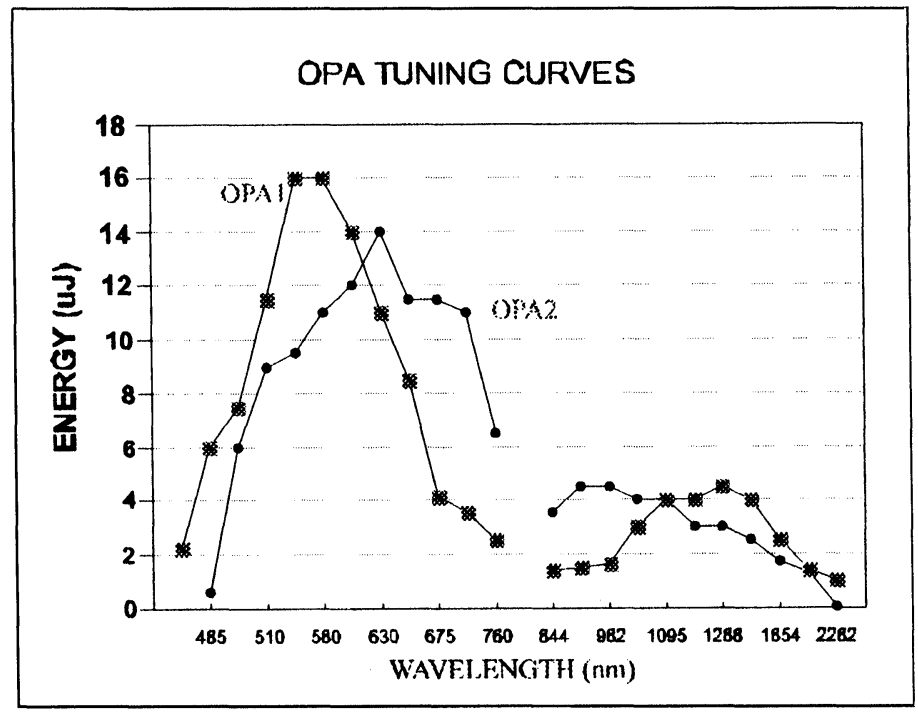

FIGURE 2 Signal and idler tuning curves of OPA1 and OPA2. 


\section{SPECTROSCOPY: TR ${ }^{3}$ SPECTROSCOPY OF RADICALS FROM THE PHOTOIONISATION OF VITAMIN E ANALOGUES}

The potential of the new system is illustrated by recent results in which ultrafast spectroscopy has been used to study the photoionisation of Trolox C, a water soluble vitamin E analogue. Scheme 1 shows how in biological membranes and lipoproteins vitamin $\mathrm{E}$ ( $\alpha$-tocopherol) is oxidised by the lipid peroxyl radical (LOO) and therefore interrupts the very damaging chain reaction of lipid peroxidation.

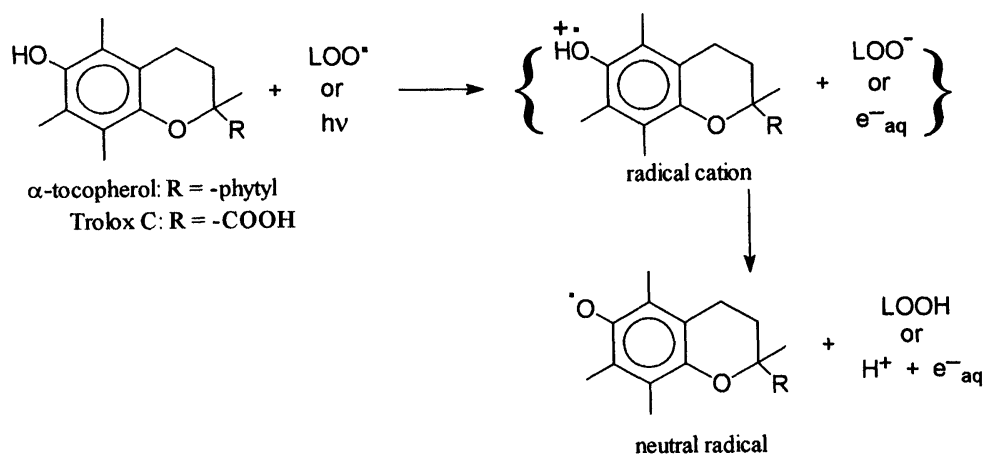

SCHEME 1

Our ultimate goal is to study details of electron transfer reactions involving vitamin $\mathrm{E}$. Photoionisation provides the most direct route to the generation and study of the neutral radical $[5,6]$ and, as in the oneelectron oxidation reaction involving $\mathrm{LOO}$, the radical cation might be expected to be a short-lived intermediate. The measured $\mathrm{pK}$ of the tocopheroxyl radical of -1.4 [7] suggests a picosecond lifetime for the radical cation after photoionisation. The kinetics of hydrated electron formation from Trolox $\mathrm{C}$ in a buffered neutral aqueous solution were observed after photoionisation with a $300 \mathrm{fs}$ pulse at $277 \mathrm{~nm}$. The curve in Figure 2 shows that the electron is formed in two steps: about half of the absorbance is formed within the risetime of the $300 \mathrm{fs}$ pulse, whereas the remaining absorbance grows in with an approximately 5 ps time constant. The electron absorbance then decays with a lifetime of about $25 \mathrm{ps}$ before reaching a value which is stable on the 
nanosecond timescale. Both the $5 \mathrm{ps}$ and 25 ps processes have $k_{\mathrm{H}} / k_{\mathrm{D}}$ of about 2.3, indicating that they are linked to the transfer of a proton [8].

We provisionally suggest that the delayed electron formation is due to deprotonation of the excited phenol which results in the autoionisation of the excited phenolate anion. The picosecond decay of the electron is proposed to be due to recombination with the geminate radical cation. Recombination is incomplete due to competition with deprotonation of the radical cation. The rate of electron decay therefore represents the combined rates of geminate radical pair separation and radical cation deprotonation. The rate of deprotonation has been estimated from observation of both the neutral and cation radicals by picosecond time-resolved resonance Raman spectroscopy, and our initial results suggest that this dominates the rate of electron decay. $\mathrm{TR}^{3}$ spectra obtained for Trolox in neutral aqueous solution are shown in Figure 4. Curves B and C show $\mathrm{TR}^{3}$ spectra obtained with pump and probe wavelengths of 283 and $425 \mathrm{~nm}$ as third and second harmonics respectively of the Ti: sapphire fundamental. At this probe wavelength both neutral and cation radicals are in resonance with extinction coefficients of the order of $4 \times 10^{3} \mathrm{dm}^{3} \mathrm{~mol}^{-1} \mathrm{~cm}^{-1}$. At $5 \mathrm{ps}$ delay between pump and probe pulses (Curve B) the spectrum consists mainly of the $1620 \mathrm{~cm}^{-1}$ peak attributable to the $\mathrm{C}=\mathrm{C}\left(\nu_{8 a}\right)$ stretching mode of the tocopheroxyl radical cation [5]. At a $1 \mathrm{~ns}$ delay (Curve $\mathrm{C}$ ) the spectrum contains two peaks representing the $\mathrm{C}-\mathrm{O}\left(\nu_{7 a}\right)$ stretch at ca. $1490 \mathrm{~cm}^{-1}$ and the $\mathrm{C}=\mathrm{C}\left(\nu_{8 a}\right)$ stretch at $1600 \mathrm{~cm}^{-1}$ of the neutral tocopheroxyl radical. Further data show that the growth of the lower frequency band is complete at $100 \mathrm{ps}$, and appears to be correlated with the electron decay observed in the transient absorption experiments such as that shown in Figure 3. Curve $A$ in Figure 4 shows the $T^{3}$ spectrum using pump and probe wavelengths of 273 and $470 \mathrm{~nm}$ respectively with a 20 ps delay. The probe wavelength in this example was obtained using the OPA system and the figure illustrates that spectra of good quality may be obtained with parametric amplification. The probe wavelength of $470 \mathrm{~nm}$ is more strongly in resonance with the radical cation $\left(\varepsilon \mathrm{ca} .10^{4} \mathrm{dm}^{3} \mathrm{~mol}^{-1} \mathrm{~cm}^{-1}\right)$. A small shift to higher frequency is discernible between the ca $1620 \mathrm{~cm}^{-1}$ band in this spectrum and that of Figure $3 \mathrm{C}$ despite the approximately similar 




FIGURE 3 Formation and decay of the transient absorbance of the hydrated electron at $830 \mathrm{~nm}$ in an aqueous solution of Trolox C containing phosphate buffer $(50 \mathrm{mM}$, $\mathrm{pH} 7$ ) on photolysis with a $300 \mathrm{fs}$ laser pulse at $277 \mathrm{~nm}$.



FIGURE 4 Picosecond $\mathrm{TR}^{3}$ spectra from neutral aqueous solution of Trolox C. Spectra B and C were obtained with pulse and probe wavelengths of 283 and $425 \mathrm{~nm}$ respectively, employing time delays between pump and probe pulses of $5 \mathrm{ps}$ (B) and $1 \mathrm{~ns}$ (C). Spectrum A was obtained using a 20 ps delay between the pump pulse at $273 \mathrm{~nm}$ and the probe pulse at $470 \mathrm{~nm}$. 
bandwidth of the probe pulse and the expected shift of $20-25 \mathrm{~cm}^{-1}$ in the $\mathrm{C}=\mathrm{C}$ band upon deprotonation of the radical cation. Further work is now in progress in order to extract kinetic information from these time resolved resonance Raman spectra.

\section{References}

[1] Matousek, P., Parker, A. W., Taday, P. F., Toner, W. T. and Towrie, M. (1996). Optics Communications, 127, 307-312.

[2] Matousek, P., Parker, A. W., Phillips, D., Toner, W. T., Towrie, M. and Scholes, G. This proceedings.

[3] Scholes, G., Matousek, P., Parker, A. W., Phillips, D. and Towrie, M. This proceedings.

[4] Toner, W. T., Matousek, P., Parker, A. W. and Towrie, M. This proceedings.

[5] Bisby, R. H. and Parker, A. W. (1991). FEBS Letters, 290, 205-208.

[6] Bisby, R. H. and Parker, A. W. (1995). Arch. Biochem. Biophys., 317, 170-178.

[7] Parker, A. W. and Bisby, R. H. (1993). J. Chem. Soc. Faraday Trans., 89, 2873-2878.

[8] Gaudel, Y. (1995). J. Mol. Liquids, 63, 1-54. 